\title{
Blick in die Zukunft: Die VDI/VDE-GMA Technologieroadmap Fertigungsmesstechnik 2020
}

Dietrich, Imkamp; Jürgen Berthold:

Anschrift(en): Carl Zeiss Industrielle Messtechnik GmbH, 73446 Oberkochen; Verein Deutscher Ingenieure e.V., VDI-Platz 1, 40468 Düsseldorf

Tel.-Nr.: +49 736420 2045; +49 211 6214-678 E-Mail: imkamp@zeiss.de; berthold@vdi.de

Die Produktionstechnik steht vor vielen Herausforderungen. Der Trend zu höherer Produktqualität und -individualität ist ungebrochen. Die Globalisierung hat die Voraussetzung für eine weltweite verknüpfte Produktion geschaffen, in der der Austausch von Informationen immer wichtiger wird. Viele von diesen Informationen besonders zum Zustand der Produkte und Produktionsprozesse werden mit Hilfe von Messtechnik, die in diesem Umfeld gemeinhin als Fertigungsmesstechnik bezeichnet wird, ermittelt. Die Fertigungsmesstechnik steht daher vor ähnlichen Herausforderungen wie die Produktionstechnik (Bild 0).

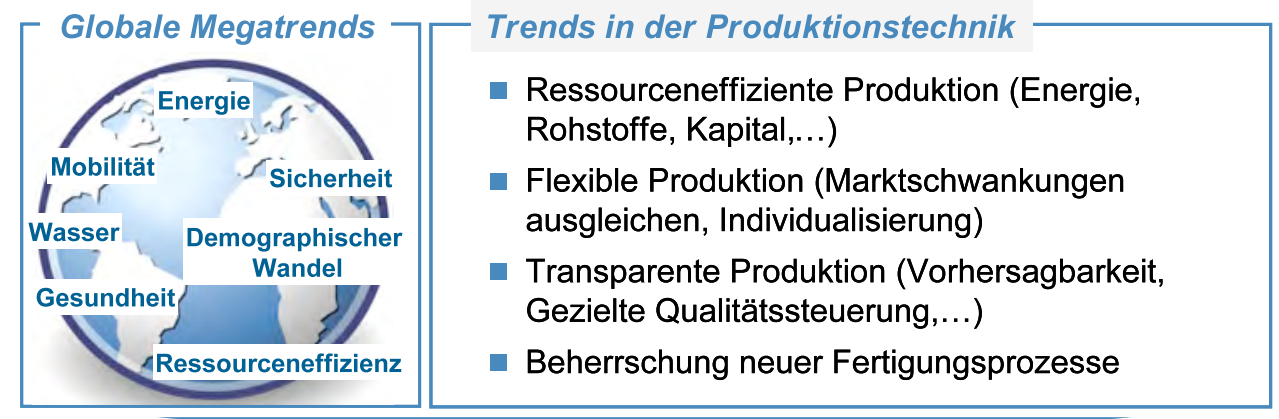

Aufgabe der

Fertigungsmesstechnik

\section{Bereitstellung valider Informationen über das Produkt zur wirtschaftlichen Absicherung der Prozess- und Produktqualität}

Bild 0: Globale Trends und Trends in der Produktionstechnik

Aus diesen Gründen hat sich eine Arbeitsgruppe der VDI/VDE-Gesellschaft Mess- und Automatisierungstechnik (GMA) der Aufgabe angenommen, einen Blick auf die Zukunft der Fertigungsmesstechnik in der industriellen Produktion zu entwickeln. Die Resultate dieser Arbeit wurden unter dem Titel „Fertigungsmesstechnik 2020 - Technologie-Roadmap für die Messtechnik in der industriellen Produktion"vom Verein Deutscher Ingenieure e.V. (VDI) veröffentlicht [1]. Zusammenfassungen wurde national [2, 3, 4] und international präsentiert [5]. In diesem Beitrag werden die wesentlichen Ergebnisse der Arbeit vorgestellt.

Anforderungen und Trends in der Fertigungsmesstechnik lassen sich mit den Begriffen „schneller“, "sicherer", „genauer" und „flexibler" beschreiben.

Mit Schnelligkeit ist einerseits die Entwicklung und Anwendung messtechnischer Verfahren gemeint, mit denen in kürzerer Zeit Informationen über die Produktqualität gewonnen werden können. Dabei kommt es weniger darauf an, Verfahren grundlegend neu zu entwickeln. Vielmehr werden viele bekannte Messprinzipien zur Nutzung in der Produktion adaptiert werden. Dabei spielen optische Verfahren eine bedeutende Rolle. Andererseits wird eine engere Integration von Messtechnik in Produktionsabläufe dazu beitragen Messergebnisse schneller zu ermitteln und effizienter zu nutzen [6]. Dadurch können Transportzeiten zu den Messeinrichtungen verringert werden oder ganz entfallen. Weiterhin stehen die Informationen aus Messungen unmittelbar in der Produktion, beispielsweise zur Realisierung von Regelkreisen, zur Verfügung. Besonders effizient kann eine Regelung durch eine automatisierte Übertragung von Daten realisiert werden $[7,8]$ (Bild 1). 
In Verbindung mit höheren Qualitätsanforderungen steigen auch die Anforderungen an die Genauigkeit der Messtechnik. Diese Entwicklung betrifft sowohl die Verfahren der Makromesstechnik [9] als auch die der Mikro- und Nanomesstechnik zur Erfassung der Produktgestalt [10] (Bild 2). In der Makromesstechnik erfordern die kleiner werdenden Toleranzen höhere Genauigkeit von Messgeräten. In der Mikromesstechnik werden höhere Genauigkeiten aufgrund der zunehmenden Miniaturisierung erforderlich. Bild 3 gibt die Größenordnung dieser Tendenzen wieder. Auch bei der Messung von Werkstoffeigenschaften und elektrischen Merkmalen steigen die Anforderungen an die Genauigkeit [11, 12]. Neben der Optimierung der Verfahren selbst gewinnen in diesem Zusammenhang die Kontrolle und die Korrektur von Umgebungseinflüssen an Bedeutung.

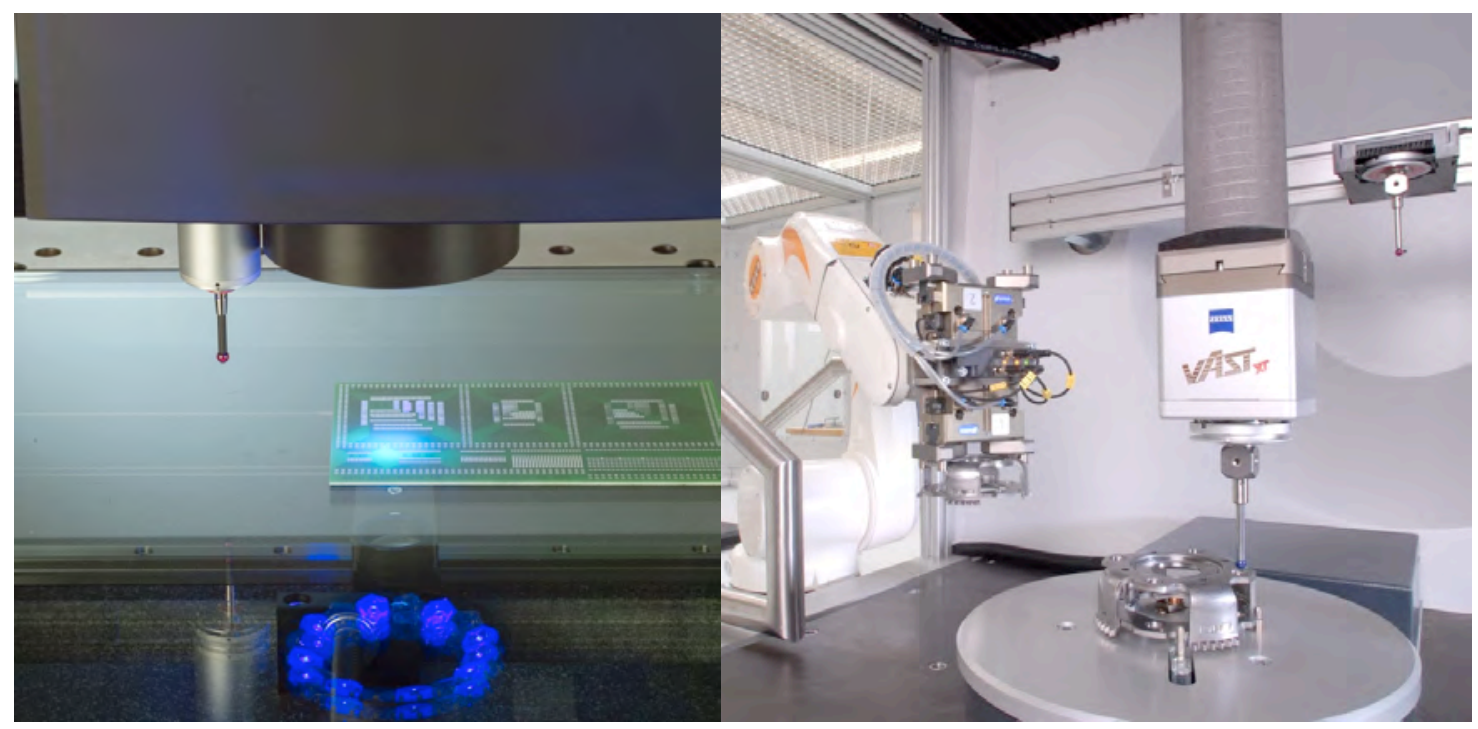

Bild 1: Schnellere Messtechnik durch Optische Kameramesstechnik und automatisierte Integration von Messtechnik in den Materialfluss durch Roboterbeladung

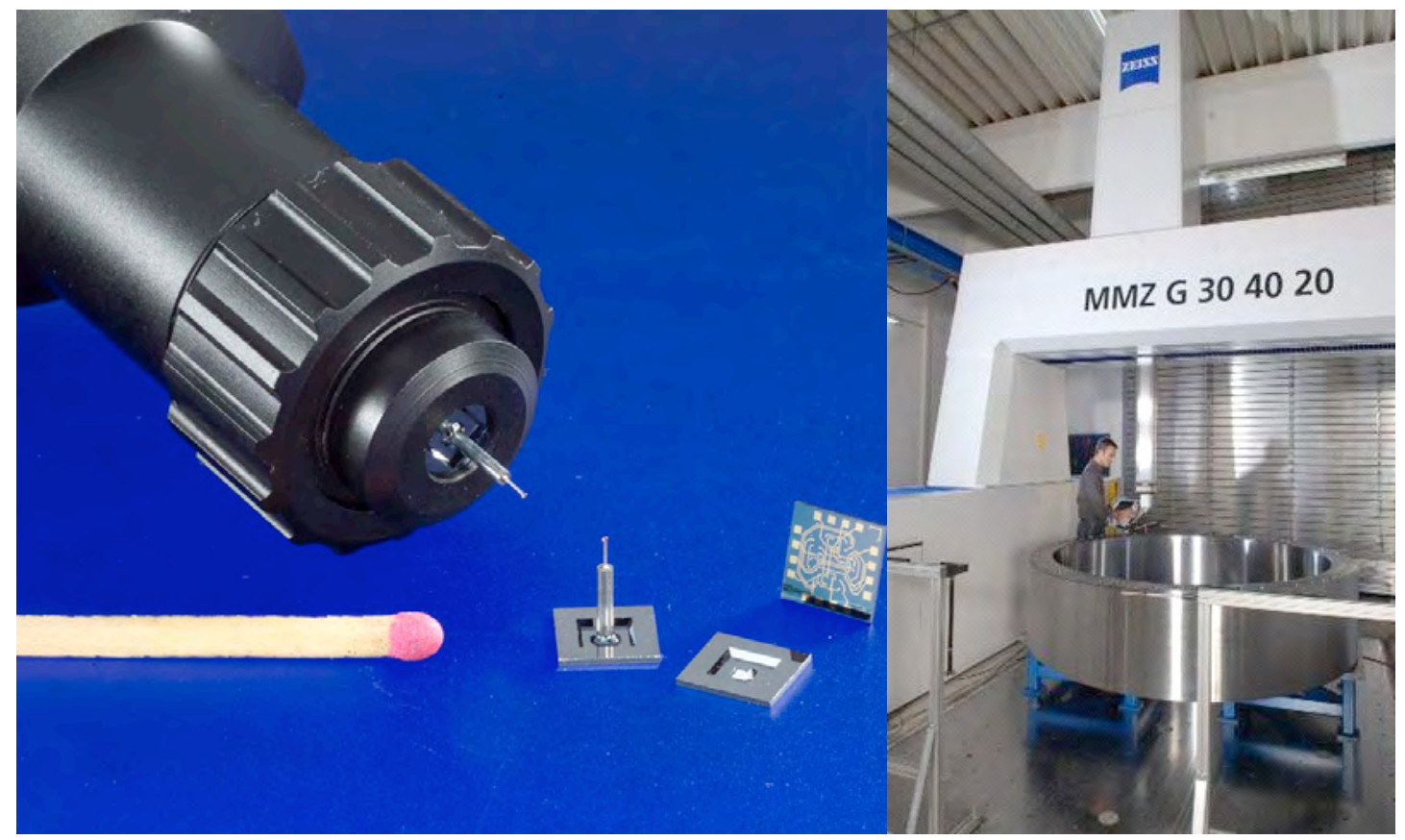

Bild 2: Genauere Messtechnik für Mikro- (Taster des Mikroteilemessgerätes F25 [13]) und Makroteile (Messung von Maschinengroßbauteilen [14]) 


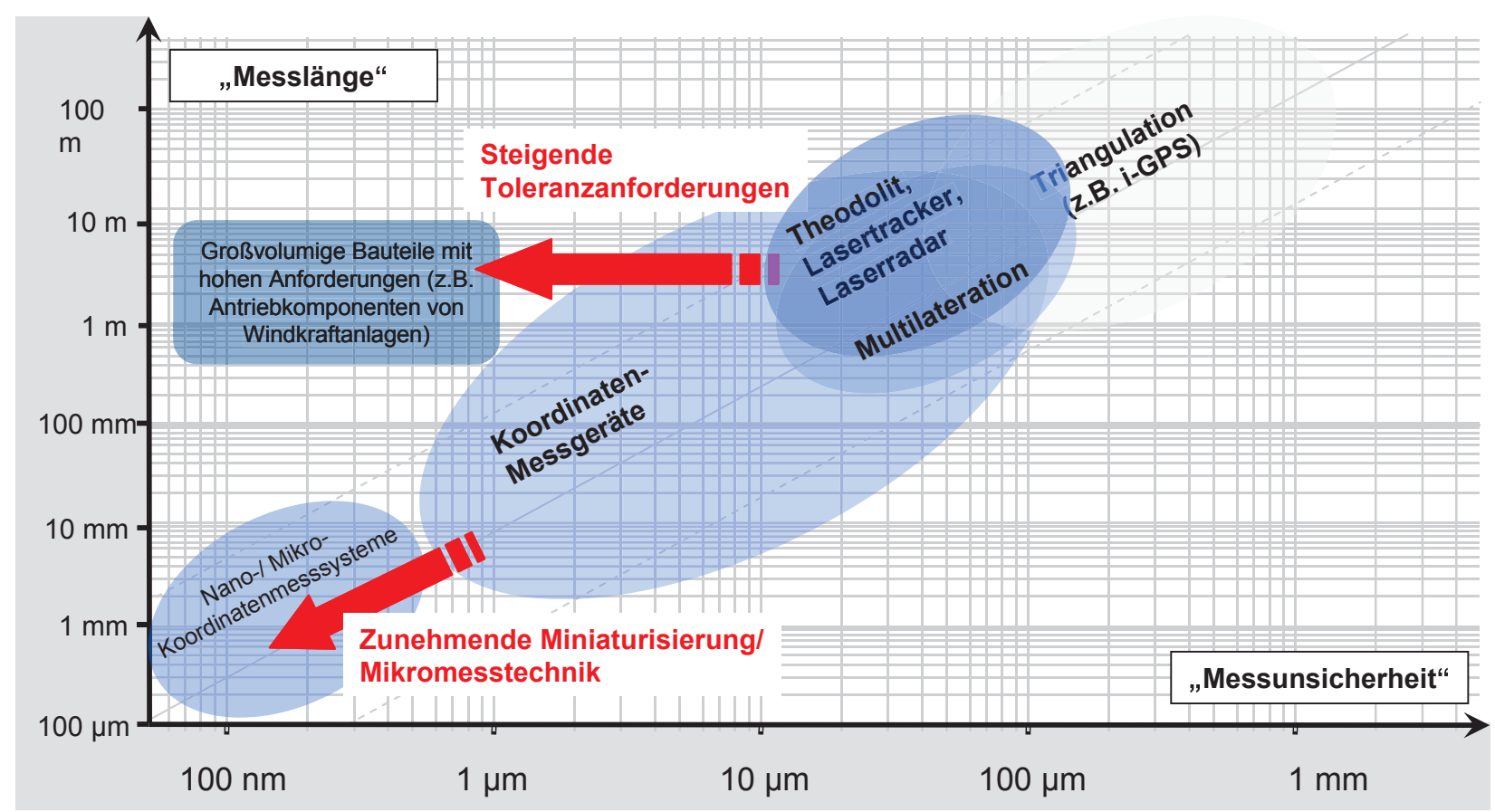

Bild 3: Tendenzen bei der Entwicklung der Genauigkeit (hier quantifiziert durch die „Messunsicherheit“) bei Geräten für die Längenmesstechnik [9]

Der Nachweis der Messunsicherheit und ihre Berücksichtigung bei der Konformitätsbewertung werden zunehmend wichtiger. Die standardisierten Vorgehensweisen zur Ermittlung der Messunsicherheit werden sich weiter etablieren. Diese werden je nach Aufgabe in unterschiedlicher Detaillierung angewendet. Für die Kalibrierung von Normalen wird mehr Aufwand bei der Unsicherheitsbestimmung zu rechtfertigen sein als bei der Prüfung einfacher Produktmerkmale. Für die Produktion werden sich vereinfachte Vorgehensweisen etablieren. Gerade bei sicherheitsrelevanten Produkten beispielsweise in der Luftfahrtindustrie und in der Medizintechnik wird ein Nachweis über die Ermittlung der Messunsicherheit und ihre Berücksichtigung bei der Prüfentscheidung zum Standard werden und die Produktsicherheit verbessern [15]. Darüber hinaus wird die rechnergestützte Simulation von Messprozessen auf Basis der Monte-Carlo Methode [16] zur Bestimmung der Messunsicherheit an Bedeutung gewinnen. Zwischenzeitlich stehen für verschiedene Messverfahren Implementierungen zumeist in Form von Prototypen zur Verfügung [17-20]. Im Bereich der Koordinatenmesstechnik (Bild 4) gibt es dazu bereits normative Festlegungen [21, 22].

Die Vielfalt der zur Messung in der Produktion eingesetzten Verfahren und damit die Flexibilität der Messtechnik nehmen rasant zu. Einerseits finden Technologien Anwendung, die ganzheitlich die Gestalt eines Produktes erfassen. Dazu gehören die Steifenprojektion und die Photogrammetrie [24]. Mit der Computertomographie lassen sich sogar Strukturen erfassen, die von außen nicht zugänglich sind [25, 26] (Bild 5). Andererseits werden vermehrt verschiedene Verfahren in Messsystemen kombiniert (Multisensorik [27] (Bild 6) und Sensorfusion [9]). Damit steigt die Flexibilität der Systeme. Allerdings steigt dadurch auch die Komplexität der Messsysteme und die Anforderungen an die Benutzer hinsichtlich Ausbildung und Aufwand bei der Vorbereitung von Messungen erhöhen sich.

Neben den zuvor beschriebenen technischen Aspekten werden in der Roadmap Fertigungsmesstechnik 2020 [1] zukünftige Entwicklung im Bereich der wirtschaftlichen Bewertung von Messtechnik und der Ausbildung sowohl an Hochschulen als auch im gewerblichen Bereich [28] dargestellt. 


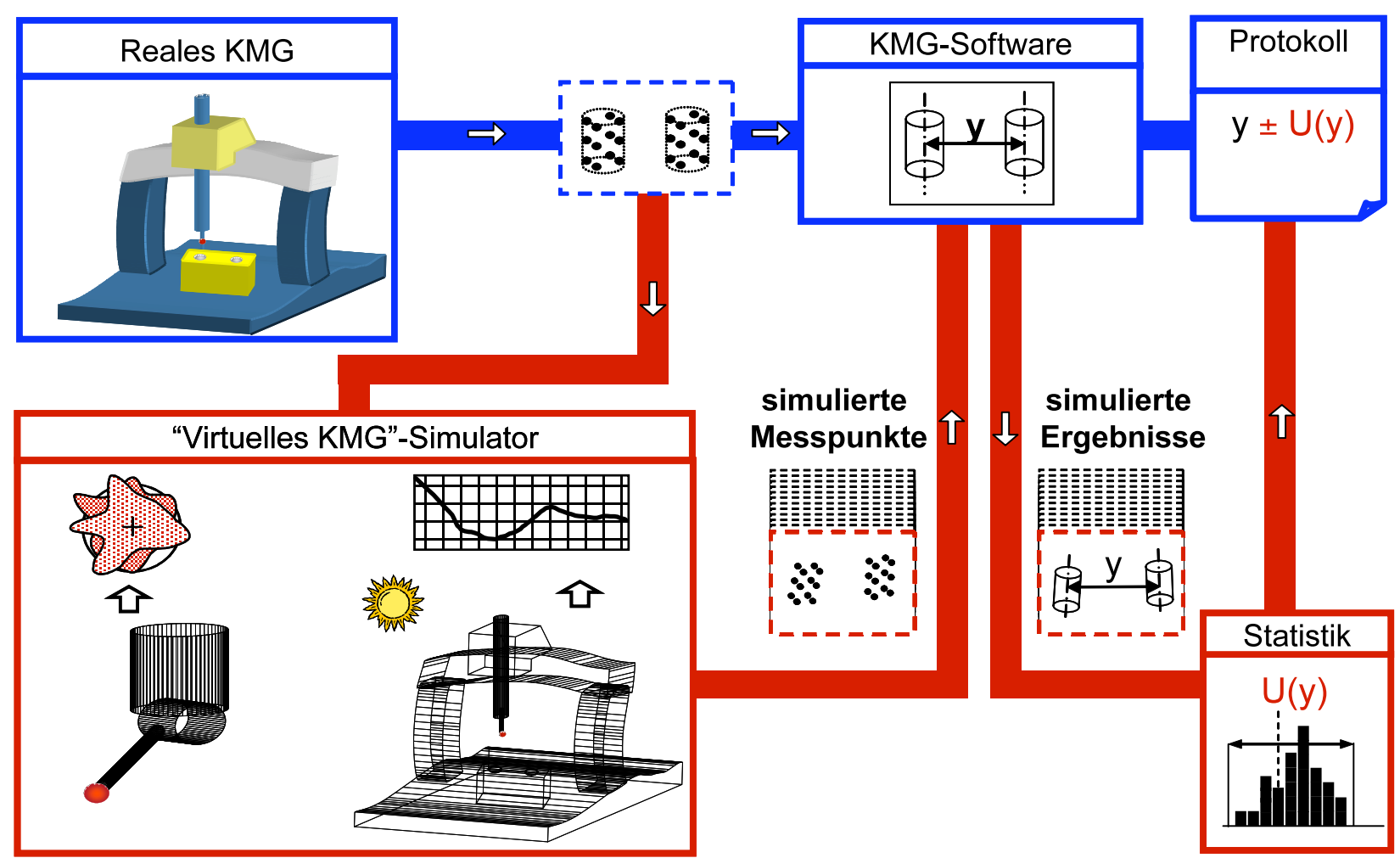

Bild 4: Sicherer: Bestimmung der Messunsicherheit von Messungen auf Koordinatenmessgeräten durch Simulation: „Virtuelles Koordinatenmessgerät“ [23] (Bildquelle: Physikalisch technische Bundesanstalt Braunschweig)

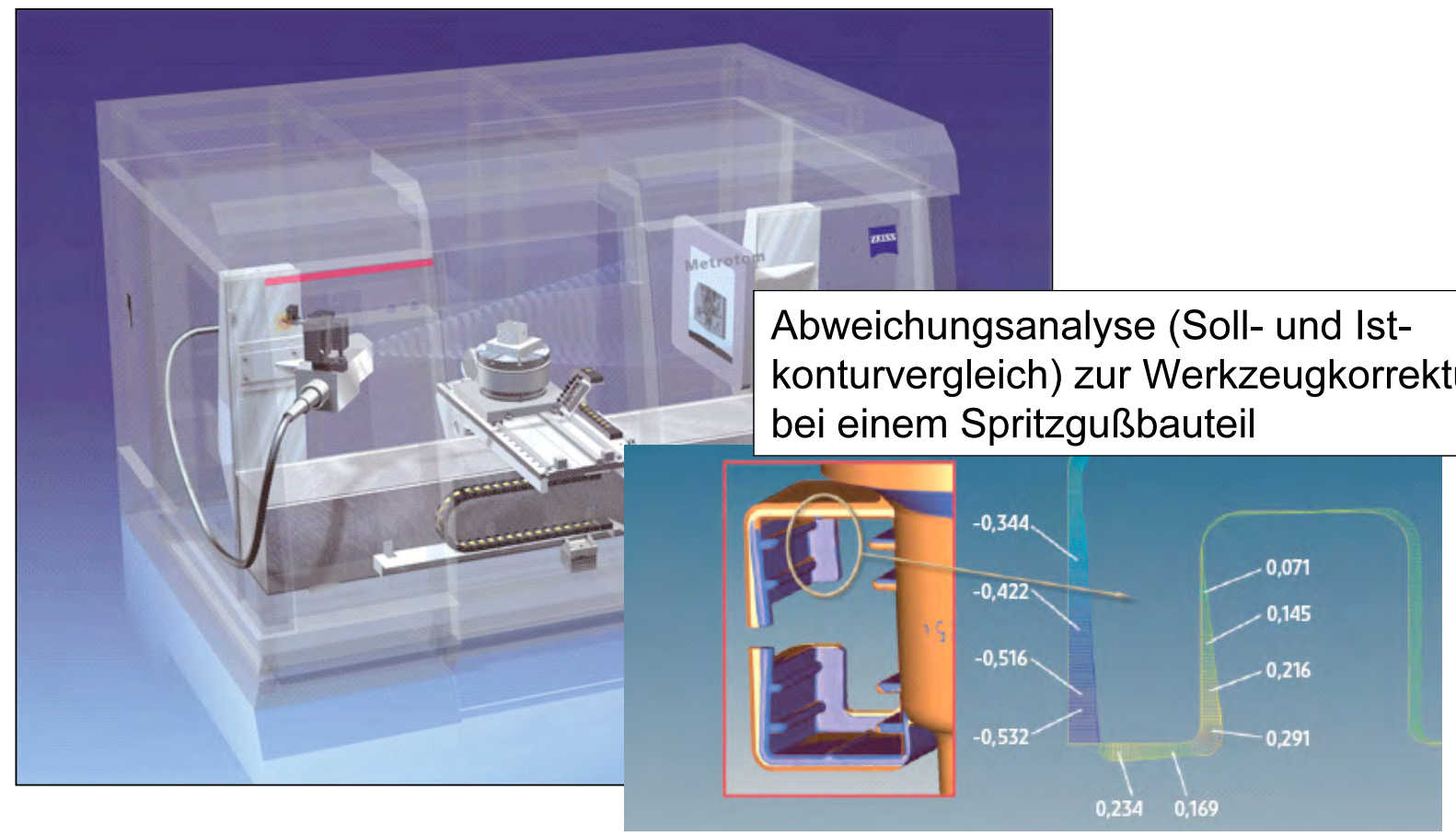

Bild 5: Flexibler: Erfassung interner Bauteilstrukturen durch Computertomographie [25] 


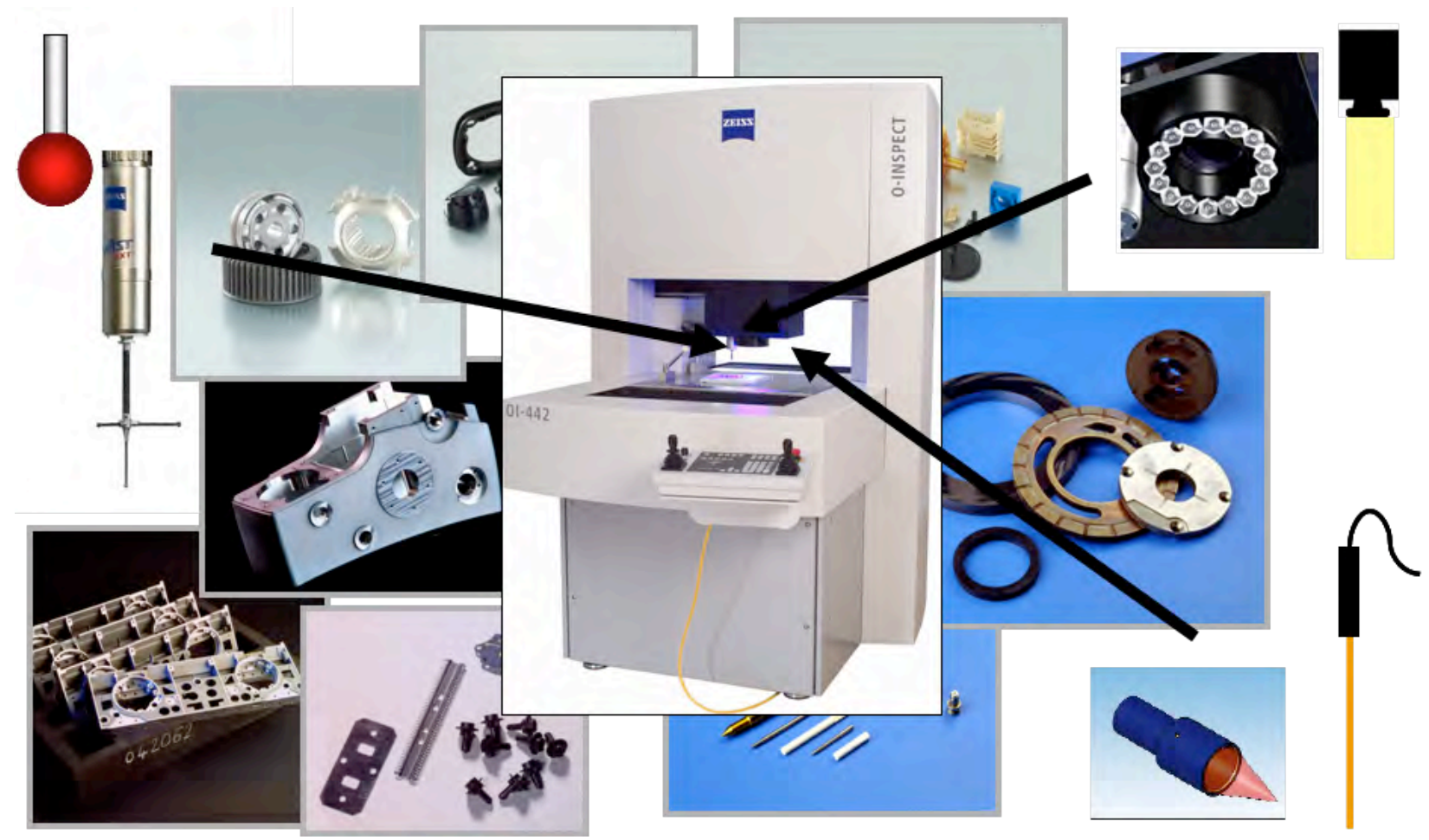

Bild 6: Flexibler: Multisensormessgerät mit taktil, scannendem Sensor (links), Kamerasensor (oben rechts) und chromatischer Weißlichtsensor als optischem Distanzsensor (unten rechts) und Beispiele für seine Anwendung

\section{Literatur:}

[1] VDI/VDE-Gesellschaft Mess- und Automatisierungstechnik (GMA) Hrsg.: Fertigungsmesstechnik 2020, Technologie-Roadmap für die Messtechnik in der industriellen Produktion, VDI Verein Deutscher Ingenieure e.V., Düsseldorf April 2011 ISBN 978-3-00-034706-1 (Internet: www.vdi.de/44080.0.html).

[2] Imkamp, D., Berthold, J.: Road to success - Der Weg der Fertigungsmesstechnik in die Zukunft, in: Qualität und Zuverlässigkeit QZ, 56. Jg., 2011, Nr. 9, S. 26-29.

[3] Schmitt, R., Imkamp, D.: Wohin entwickelt sich die Fertigungsmesstechnik? - Roadmap Fertigungsmesstechnik 2020 der VDI/VDE-Gesellschaft Mess- und Automatisierungstechnik (GMA), in: atp edition, Automatisierungstechnische Praxis, Nr. 6, 2011.

[4] Imkamp, D., Berthold, J.: Schneller, sicherer, genauer - Roadmap Fertigungsmesstechnik 2020 (Teil 1), in: Qualität und Zuverlässigkeit QZ, 54. Jg., 2009, Nr. 5, S. 36-39.

[5] Schmitt, R., Nisch, S., Heizmann, M., Bosse, H., Imkamp, D.: Production Metrology - Future Trends and Challenges, in: Proceedings of the 10th International Symposium on Measurement Technology and Intelligent Instruments (ISMTII-2011) Daejeon, S. Korea 29th June - 2nd July, 2011.

[6] Imkamp, D., Frankenfeld, T.: Schnittstellen zur informationstechnischen Integration von Geräten der Fertigungsmesstechnik in die automatisierte Produktion, in: Tagungsband zur Automation 2009, 16.-17. Juni 2009, Baden-Baden, VDI Verlag Düsseldorf 2009.

[7] Heizmann, M., Beyerer, J., Puente León, F.: Mehr Wissen durch Fusion von Sensordaten Roadmap Fertigungsmesstechnik 2020 (Teil 2), Qualität und Zuverlässigkeit QZ, 54. Jg., 2009, Nr. 6, S. 35-39.

[8] Pfeifer, T., Imkamp, D.: Koordinatenmesstechnik und CAx- Anwendungen in der Produktion Grundlagen, Schnittstellen und Integration, Carl Hanser Verlag, München 2004.

[9] Schmitt, R., Jatzkowski, P., Nisch, S., Imkamp, D.: Größer, genauer und integrierter - Roadmap Fertigungsmesstechnik 2020 (Teil 5), in: Qualität und Zuverlässigkeit QZ, 54. Jg., 2009, Nr. 9, S. 31-33.

[10] Bosse, H., Koenders, L., Schmitt, R.: Von Mikro zu Nano - Roadmap Fertigungsmesstechnik 2020 (Teil 3), Qualität und Zuverlässigkeit QZ, 54. Jg., 2009, Nr. 7, S. 28-31. 
[11] Frenz, H., Schenuit, E.: Sinkende Toleranzschwelle - Roadmap Fertigungsmesstechnik 2020 (Teil 7), Qualität und Zuverlässigkeit QZ, 54. Jg., 2009, Nr. 11, S. 47-49.

[12] Naß, M., Berthold, J.: Basis neuer Messtechnologien - Roadmap Fertigungsmesstechnik 2020 (Teil 8), Qualität und Zuverlässigkeit QZ, 55. Jg. 2010, Nr. 1, S. 53-55.

[13] Wiedmann, W. K., Imkamp, D., Bader, F.: Mikroteilemessgerät F25 - Einsatzbereiche und Anwendungserfahrung in: Tagungsband (VDI-Bericht 2133) zur 4. Fachtagung „Metrologie in der Mikro- und Nanotechnik 2011 -Messprinzipien - Messgeräte - Anwendungen“, 25. und 26. Oktober 2011 in Erlangen, VDI Wissensforum GmbH, Düsseldorf 2011.

[14] Daniel, H., Dussling, J., Brandstetter, T.: Koordinatenmesstechnik im Aufwind, Qualitätssicherung bei Windenergieanlagen, in: Quality Engineering, Oktober 2009, S. 30f., und in: mav, Oktober 2009, S. 110-113., Konradin Verlag, Leinfelden-Echterdingen.

[15] Imkamp, D., Sommer, K.-D.: Für eine sichere Fertigung, Roadmap Fertigungsmesstechnik 2020 (Teil 4), in: Qualität und Zuverlässigkeit QZ, 54. Jg., 2009, Nr. 8, S. 31-33.

[16] JGCM 101:2008 Evaluation of measurement data - Supplement 1 to the "Guide to the expression of uncertainty in measurement" - Propagation of distributions using a Monte Carlo method, JCGM (Joint Committee for Guides in Metrology) 2008 (Internet: http://www.bipm.org/en/publications/guides/gum.html).

[17] Schwenke, H.: Abschätzung von Messunsicherheiten durch Simulation an Beispielen der Fertigungsmesstechnik (Dissertation), PTB-Bericht F36, 1999.

[18] Bai, A., Bitte, F., Pfeifer, T.: Der Einsatz von Simulationen zur Bestimmung der Messunsicherheit von Interferometern (Assessment of the Measurement Uncertainty of Interferometers by Means of Simulation), in: tm - Technisches Messen, Oldenbourg Industrieverlag, 69 (2002) 5 S. 27-32.

[19] Hiller, J.: Abschätzung von Unsicherheiten beim dimensionellen Messen mit industrieller RöntgenComputertomographie durch Simulation, Dissertation, Universität Freiburg 2011.

[20] Schmitt, R., Fritz, P., Jatzkowski, P., Lose, J., Koerfer, F., Wendt, K.: Abschätzung der Messunsicherheit komplexer Messsysteme mittels statistischer Simulation durch den Hersteller, in: VDI/VDE-Gesellschaft Meß- und Automatisierungstechnik -GMA (Hrsg.): Messunsicherheit praxisgerecht bestimmen, Tagungsbericht: 4. Fachtagung Messunsicherheit. 12. und 13. November 2008 in Erfurt, Düsseldorf: VDI Wissensforum, 2008.

[21] ISO/TS 15530-4 Geometrical Product Specifications (GPS) - Coordinate measuring machines (CMM): Technique for determining the uncertainty of measurement - Part 4: Evaluating taskspecific measurement uncertainty using simulation Ausgabe: 2008-06.

[22] VDI/VDE-Richtlinie 2617 Blatt 7 (part 7) Genauigkeit von Koordinatenmessgeräten - Kenngrößen und deren Prüfung - Ermittlung der Unsicherheit von Messungen auf Koordinatenmessgeräten durch Simulation (English: Accuracy of coordinate measuring machines - Parameters and their checking - Estimation of measurement uncertainty of coordinate measuring machines by means of simulation) April 2006.

[23] Wäldele, F., Franke, M., Härtig, F., Schwenke, H., Trapet, E.: So genau wie nötig messen, Messunsicherheit auf Koordinatenmessgeräten automatisch ermitteln, QZ, Qualität und Zuverlässigkeit, Carl Hanser Verlag, München Jg. 50 (2005), Nr. 3, S. 58-61.

[24] Bauer, N. (Hrsg.): Leitfaden zu Grundlagen und Anwendungen der optischen 3D-Messtechnik, Vision 6, Fraunhofer Allianz Vision, Erlangen 2003.

[25] Benninger, R., Bleicher, M., Berthold, J.: Mit Röntgenblick zum Allrounder - Roadmap Fertigungsmesstechnik 2020 (Teil 6), Qualität und Zuverlässigkeit QZ, 54. Jg., 2009, Nr. 10, S. 4447.

[26] Imkamp, D., Lettenbauer, H., Georgi, B.: Computertomographie für die dimensionelle Fertigungsmesstechnik - Status und Potentiale, in: Vorträge der 13. ITG/GMA-Fachtagung, Sensoren und Messsysteme in Freiburg/Breisgau, Germany, 13./14. März 2006, VDE Verlag $\mathrm{GmbH}$, Berlin 2006.

[27] Imkamp, D., Vizcaino-Hoppe, M.: Mehr als die Summe der Sensoren - Optische Sensoren für Multisensor-Koordinatenmessgeräte, in: Tagungsband zur VDI Tagung Optische Messung technischer Oberflächen, 09.-10. Oktober 2007, Hannover, (VDI Bericht 1996), VDI Verlag Düsseldorf 2007.

[28] Wäldele, F.: Die Wissenstankstelle für Fertigungsmesstechnik, 10 Jahre AUKOM in: Quality Engineering, Konradin Verlag, Leinfelden-Echterdingen, Nr. 6, 2011, S. 27ff. (Internet: www.aukomev.de) 